\title{
Invariance analysis and exact solutions of some sixth-order difference equations
}

\author{
Darlison Nyirenda, Mensah Folly-Gbetoula* \\ School of Mathematics, University of the Witwatersrand, 2050, Johannesburg, South Africa.
}

Communicated by X.-J. Yang

\begin{abstract}
We perform a full Lie point symmetry analysis of difference equations of the form

$$
u_{n+6}=\frac{u_{n} u_{n+4}}{u_{n+2}\left(A_{n}+B_{n} u_{n} u_{n+4}\right)},
$$

where the initial conditions are non-zero real numbers. Consequently, we obtain four non-trivial symmetries. Eventually, we get solutions of the difference equation for random sequences $\left(A_{n}\right)$ and $\left(B_{n}\right)$. This work is a generalization of a recent result by Khaliq and Elsayed [A. Khaliq, E. M. Elsayed, J. Nonlinear Sci. Appl., 9 (2016), 1052-1063]. (C)2017 All rights reserved.
\end{abstract}

Keywords: Difference equation, symmetry, group invariant solutions.

2010 MSC: 39A10, 39A99, 39A13.

\section{Introduction}

In the past years, after the work of Lie [12] on differential equations, several researchers became interested in symmetries. Lie studied the group of mappings which leaves the differential equations invariant. The notion of symmetry is linked to conservation laws and the link between them has sparked great interest in researchers following the work of Noether [14]. It is a clear fact that as long as the symmetries and first integrals are related through the condition of invariance, one can execute the double reduction of the differential equations $[13,16]$. The symmetry method has been used to find traveling wave solutions. For more on traveling waves, refer to [2, 17, 18] and [19]. The idea of symmetry method has been extended to difference equations and we refer the reader to [3-6, 11, 15] and references therein. Hydon [6] came up with a symmetry based procedure which makes it possible for one to find solutions of difference equations without trial and error. Much as Hydon, in his book [6], put emphasis on difference equations of second order, his algorithm works for any order. Unfortunately in such higher-order cases, computations are usually cumbersome and so certain assumptions are made to ease the computation. When this method is employed, we expect single solutions with fewer constraints on initial conditions.

\footnotetext{
${ }^{*}$ Corresponding author

Email addresses: Darlison.Nyirenda@wits.ac.za (Darlison Nyirenda), Mensah.Folly-Gbetoula@wits.ac.za (Mensah Folly-Gbetoula )
}

doi:10.22436/jnsa.010.12.11 
Our work is inspired by the results of Khaliq and Elsayed [9] where the following difference equation was studied

$$
x_{n+1}=\frac{x_{n-1} x_{n-5}}{x_{n-3}\left( \pm 1 \pm x_{n-1} x_{n-5}\right)}
$$

where $x_{-5}, x_{-4}, x_{-3}, x_{-2}, x_{-1}$ and $x_{0}$ are initial conditions and are positive real numbers. It is clear that (1.1) is specific case of a general form

$$
x_{n+1}=\frac{x_{n-1} x_{n-5}}{x_{n-3}\left(a_{n}+b_{n} x_{n-1} x_{n-5}\right)},
$$

where $\left(a_{n}\right)$ and $\left(b_{n}\right)$ are real sequences. Our goal is to use a symmetry based method to solve the more general difference equation (1.2). For definiteness, we study the equation

$$
u_{n+6}=\frac{u_{n} u_{n+4}}{u_{n+2}\left(A_{n}+B_{n} u_{n} u_{n+4}\right)},
$$

instead.

For related work, refer to $[1,7,8]$ and [10].

\section{Preliminaries}

In this section, we follow definitions and notation from [6].

Definition 2.1. A parameterized set of point transformations,

$$
\Gamma_{\varepsilon}: x \mapsto \hat{x}(x ; \varepsilon),
$$

where $x=x_{i}, i=1, \cdots, p$ are continuous variables, is a one-parameter local Lie group of transformations if the following conditions are satisfied:

1. $\Gamma_{0}$ is the identity map if $\hat{x}=x$ when $\varepsilon=0$.

2. $\Gamma_{\mathrm{a}} \Gamma_{\mathrm{b}}=\Gamma_{\mathrm{a}+\mathrm{b}}$ for every $\mathrm{a}$ and $\mathrm{b}$ sufficiently close to 0 .

3. Each $\hat{x_{i}}$ can be represented as a Taylor series (in a neighborhood of $\varepsilon=0$ that is determined by $x$ ), and therefore

$$
\hat{x_{i}}(x: \varepsilon)=x_{i}+\varepsilon \xi_{i}(x)+O\left(\varepsilon^{2}\right), \quad i=1, \cdots, p .
$$

Given the p-th-order difference equation

$$
u_{n+p}=w\left(u_{n}, \cdots, u_{n+p-1}\right)
$$

for some function $\omega$. We confine ourselves to symmetries where $\hat{u}_{n}$ depends on $n$ and $u_{n}$ only. Suppose that the point transformations take the following shape:

$$
\hat{n}=n, \quad \hat{u}_{n}=u_{n}+\epsilon Q\left(n, u_{n}\right) .
$$

Thus the symmetry condition is defined as

$$
\hat{u}_{n+p}=\omega\left(n, \hat{u}_{n}, \hat{u}_{n+1}, \cdots, \hat{u}_{n+p-1}\right),
$$

whenever (2.1) is true. Performing a substitution of Lie point symmetries (2.2) into the condition (2.3) results in the symmetry condition

$$
\mathrm{S}^{(\mathrm{p})} \mathrm{Q}-\mathrm{X} \omega=0,
$$

whenever (2.1) holds, in which $S$ is the shift operator, that is, $S: n \mapsto n+1$. With the following known infinitesimal symmetry generator

$$
X=Q\left(n, u_{n}\right) \frac{\partial}{\partial u_{n}}+S Q\left(n, u_{n}\right) \frac{\partial}{\partial u_{n+1}}+\cdots+S^{n+p-1} Q\left(n, u_{n}\right) \frac{\partial}{\partial u_{n+p-1}},
$$

it is important to take the canonical coordinate into consideration

$$
s_{n}=\int \frac{d u_{n}}{Q\left(n, u_{n}\right)} \text {. }
$$




\section{Symmetries and exact solutions}

Considering the sixth-order difference equation of the form

$$
u_{n+6}=\frac{u_{n} u_{n+4}}{u_{n+2}\left(A_{n}+B_{n} u_{n} u_{n+4}\right)} .
$$

Imposing the symmetry condition (2.4) and with a bit of simplification, we get

$$
\begin{aligned}
Q\left(n+6, u_{n+6}\right)-\frac{A_{n} u_{n}}{u_{n+2}\left(B_{n} u_{n} u_{n+4}+A_{n}\right)^{2}} Q\left(n+4, u_{n+4}\right) \\
\quad+\frac{u_{n} u_{n+4}}{u_{n+2}\left(B_{n} u_{n} u_{n+4}+A_{n}\right)} Q\left(n+2, u_{n+2}\right)-\frac{A_{n} u_{n+4}}{u_{n+2}\left(B_{n} u_{n} u_{n+4}+A_{n}\right)^{2}} Q\left(n, u_{n}\right)=0 .
\end{aligned}
$$

In solving for the characteristic function, differentiate (3.2) with respect to $u_{n}$ (keeping $\omega$ fixed) and consider $u_{n+2}$ as a function of $u_{n}, u_{n+4}$ and $\omega$. This yields

$$
\begin{aligned}
B_{n} u_{n+4} u_{n} u_{n+2} \frac{\partial}{\partial u_{n+2}} Q\left(n+2, u_{n+2}\right)-B_{n} u_{n+4} u_{n} u_{n+2} \frac{\partial}{\partial u_{n}} Q\left(n, u_{n}\right) \\
\quad-B_{n} u_{n+4} u_{n} Q\left(n+2, u_{n+2}\right)+B_{n} u_{n} u_{n+2} Q\left(n+4, u_{n+4}\right) \\
+2 B_{n} u_{n+4} u_{n+2} Q\left(n, u_{n}\right)+A_{n} u_{n+2} \frac{\partial}{\partial u_{n+2}} Q\left(n+2, u_{n+2}\right) \\
-A_{n} u_{n+2} \frac{\partial}{\partial u_{n}} Q\left(n, u_{n}\right)-A_{n} Q\left(n+2, u_{n+2}\right)+\frac{A_{n} u_{n+2}}{u_{n}} Q\left(n, u_{n}\right)=0 .
\end{aligned}
$$

We now proceed by differentiating (3.3) with respect to $u_{n}$ twice (keeping $u_{n+2}$ fixed) and obtain

$$
\begin{gathered}
-B_{n} u_{n+4} u_{n} \frac{\partial^{3}}{\partial u_{n}{ }^{3}} Q\left(n, u_{n}\right)-A_{n} \frac{\partial^{3}}{\partial u_{n}{ }^{3}} Q\left(n, u_{n}\right)+\frac{A_{n}}{u_{n}} \frac{\partial^{2}}{\partial u_{n}{ }^{2}} Q\left(n, u_{n}\right) \\
-\frac{2 A_{n}}{u_{n}^{2}} \frac{\partial}{\partial u_{n}} Q\left(n, u_{n}\right)+\frac{2 A_{n}}{u_{n}^{3}} Q\left(n, u_{n}\right)=0 .
\end{gathered}
$$

The equation above is solved by separation of variables in powers of shifts of $u_{n}$. Thus we have

$$
\left\{\begin{array}{l}
u_{n+4}:-B_{n} u_{n} Q^{(3)}=0 \\
1:-A_{n} Q^{(3)}+\frac{A_{n}}{u_{n}} Q^{(2)}-\frac{2 A_{n}}{u_{n}{ }^{2}} Q^{(1)}+\frac{2 A_{n}}{u_{n}{ }^{3}} Q=0
\end{array}\right.
$$

which has the solution

$$
Q=Q\left(n, u_{n}\right)=\alpha_{n} u_{n}^{2}+\beta_{n} u_{n}
$$

where $\alpha_{n}$ and $\beta_{n}$ are some functions of $n$. We then substitute (3.4) in (3.2) and split the result so that the following is obtained:

$$
\left\{\begin{aligned}
u_{n+4}{ }^{2} u_{n}{ }^{2} u_{n+2}{ }^{2}: & B_{n} \alpha_{n+2}=0, \\
u_{n+4}{ }^{2} u_{n}{ }^{2} u_{n+2}: & B_{n}\left(\beta_{n+2}+\beta_{n+6}\right)=0, \\
u_{n+4}{ }^{2} u_{n} u_{n+2}: & A_{n} \alpha_{n+4}=0, \\
u_{n+4} u_{n}{ }^{2} u_{n+2}: & A_{n} \alpha_{n}=0, \\
u_{n+4} u_{n} u_{n+2}: & A_{n} \alpha_{n+2}=0, \\
u_{n+4}{ }^{2} u_{n}{ }^{2}: & \alpha_{n+6}=0, \\
u_{n+4} u_{n} u_{n+2}: & A_{n}\left(-\beta_{n}+\beta_{n+2}-\beta_{n+4}+\beta_{n+6}\right)=0 .
\end{aligned}\right.
$$


The system above (3.5) has solutions

$$
\begin{aligned}
& \alpha_{n}=0, \\
& \beta_{n}=\left(-\frac{\sqrt{2}}{2}-i \frac{\sqrt{2}}{2}\right)^{n} c_{1}+\left(-\frac{\sqrt{2}}{2}+i \frac{\sqrt{2}}{2}\right)^{n} c_{2}+\left(\frac{\sqrt{2}}{2}-i \frac{\sqrt{2}}{2}\right)^{n} c_{3}+\left(\frac{\sqrt{2}}{2}+i \frac{\sqrt{2}}{2}\right)^{n} c_{4}
\end{aligned}
$$

for some arbitrary constants $c_{i}, i=1, \cdots, 4$. So we obtain four characteristics and their corresponding generators are as follows:

$$
\begin{aligned}
X_{1}= & (-1)^{n} \beta^{n} u_{n} \partial u_{n}-(-1)^{n} \beta^{n+1} u_{n+1} \partial_{u_{n+1}}+(-1)^{n} \beta^{n+2} u_{n+2} \partial_{u_{n+2}} \\
& -(-1)^{n} \beta^{n+3} u_{n+3} \partial_{u_{n+3}}+(-1)^{n} \beta^{n+4} u_{n+4} \partial_{u_{n+4}}-(-1)^{n} \beta^{n+5} u_{n+5} \partial_{u_{n+5}} \\
X_{2}= & (-1)^{n} \bar{\beta}^{n} u_{n} \partial u_{n}-(-1)^{n} \bar{\beta}^{n+1} u_{n+1} \partial_{u_{n+1}}+(-1)^{n} \bar{\beta}^{n+2} u_{n+2} \partial_{u_{n+2}} \\
& -(-1)^{n} \bar{\beta}^{n+3} u_{n+3} \partial_{u_{n+3}}+(-1)^{n} \bar{\beta}^{n+4} u_{n+4} \partial_{u_{n+4}}-(-1)^{n} \bar{\beta}^{n+5} u_{n+5} \partial_{u_{n+5}} \\
X_{3}= & \bar{\beta}^{n} u_{n} \partial u_{n}+\bar{\beta}^{n+1} u_{n+1} \partial_{u_{n+1}}+\bar{\beta}^{n+2} u_{n+2} \partial_{u_{n+2}}+\bar{\beta}^{n+3} u_{n+3} \partial_{u_{n+3}} \\
& +\bar{\beta}^{n+4} u_{n+4} \partial_{u_{n+4}}+\bar{\beta}^{n+5} u_{n+5} \partial_{u_{n+5}} \\
X_{4}= & \beta^{n} u_{n} \partial u_{n}+\beta^{n+1} u_{n+1} \partial_{u_{n+1}}+\beta^{n+2} u_{n+2} \partial_{u_{n+2}}+\beta^{n+3} u_{n+3} \partial_{u_{n+3}} \\
& +\beta^{n+4} u_{n+4} \partial_{u_{n+4}}+\beta^{n+5} u_{n+5} \partial_{u_{n+5}}
\end{aligned}
$$

in which $\beta=\exp \left(\frac{\pi i}{4}\right)$.

Now, utilizing $X_{4}$, we introduce the canonical coordinate

$$
s_{n}=\int \frac{d u_{n}}{\beta^{n} u_{n}}=\bar{\beta}^{n} \ln \left|u_{n}\right| \text {. }
$$

Set

$$
\tilde{V}_{n}=\beta^{n+4} s_{n+4}+\beta^{n} s_{n}
$$

and

$$
\left|\mathrm{V}_{\mathrm{n}}\right|=\exp \left\{-\tilde{\mathrm{V}}_{\mathrm{n}}\right\}
$$

i.e., $V_{n}= \pm 1 /\left(u_{n} u_{n+4}\right)$ but we choose $V_{n}=1 /\left(u_{n} u_{n+4}\right)$. Using (3.1), one can check that

$$
V_{n+2}=A_{n} V_{n}+B_{n}
$$

and thus

$$
V_{2 n+k}=V_{k}\left(\prod_{k_{1}=0}^{n-1} A_{2 k_{1}+k}\right)+\sum_{l=0}^{n-1}\left(B_{2 l+k} \prod_{k_{2}=l+1}^{n-1} A_{2 k_{2}+k}\right), \quad k=0,1
$$

We have that

$$
\begin{aligned}
\left|u_{n}\right|= & \exp \left\{\beta^{n} s_{n}\right\} \\
= & \exp \beta^{n}\left(c_{6}+i^{n} c_{7}+(-1)^{n} c_{8}+(-i)^{n} c_{9}+\frac{1}{4}\left[\sum_{k_{1}=0}^{n-1} r_{k_{1}}+i^{n} \sum_{k_{2}=0}^{n-1}(-i)^{k_{2}} r_{k_{2}}\right.\right. \\
& \left.\left.+(-1)^{n} \sum_{k_{3}=0}^{n-1}(-1)^{k_{3}} r_{k_{3}}+(-i)^{n} \sum_{k_{4}=0}^{n-1}(i)^{k_{4}} r_{k_{4}}+\right]\right) \\
= & \exp \beta^{n}\left(c_{6}+i^{n} c_{7}+(-1)^{n} c_{8}+(-i)^{n} c_{9}+\frac{1}{4}\left[\sum_{k_{1}=0}^{n-1} \bar{\beta}^{k_{1}} \ln \left|V_{k_{1}}\right|+i^{n} \sum_{k_{2}=0}^{n-1}(-i)^{k_{2}} \bar{\beta}^{k_{2}} \ln \left|V_{k_{2}}\right|\right.\right. \\
& \left.\left.+(-1)^{n} \sum_{k_{3}=0}^{n-1}(-1)^{k_{3}} \bar{\beta}^{k_{3}} \ln \left|V_{k_{3}}\right|+(-i)^{n} \sum_{k_{4}=0}^{n-1}(i)^{k_{4}} \bar{\beta}^{k_{4}} \ln \left|V_{k_{4}}\right|\right]\right),
\end{aligned}
$$




$$
\left|u_{n}\right|=\exp \left(\frac{1}{2} \sum_{k_{1}=0}^{n-1}\left[1+(-1)^{n+k_{1}}\right] \operatorname{Re}\left(\gamma\left(n, k_{1}\right)\right) \ln \left|v_{k_{1}}\right|+\beta^{n} c_{6}+(-\bar{\beta})^{n} c_{7}+(-1)^{n} \beta^{n} c_{8}+\bar{\beta}^{n} c_{9}\right),
$$

where $V_{k}$ is given in (3.6) with $\gamma(n, k)=\beta^{n} \bar{\beta}^{k}$. Observe that $c_{6}, c_{7}, c_{8}$ and $c_{9}$ satisfy

$$
\begin{aligned}
& c_{6}+c_{7}+c_{8}+c_{9}=\ln \left|u_{0}\right|, \\
& \beta c_{6}-\bar{\beta} c_{7}-\beta c_{8}+\bar{\beta} c_{9}=\ln \left|u_{1}\right|, \\
& \beta^{2} c_{6}+\bar{\beta}^{2} c_{7}+\beta^{2} c_{8}+\bar{\beta}^{2} c_{9}=\ln \left|u_{2}\right|, \\
& \beta^{3} c_{6}-\bar{\beta}^{3} c_{7}-\beta^{3} c_{8}+\bar{\beta}^{3} c_{9}=\ln \left|u_{3}\right| .
\end{aligned}
$$

Equations (3.7), (3.8), (3.9), (3.10), (3.11), (3.12) give the solutions of (3.1).

The function $\gamma(n, k)=\beta^{n} \bar{\beta}^{k}$ is such that

$$
\begin{aligned}
& \gamma(0,1)=\bar{\beta}, \quad \gamma(0,2)=-i, \quad \gamma(0,3)=-\beta, \quad \gamma(1,0)=\beta, \quad \gamma(1,2)=\bar{\beta}, \quad \gamma(1,3)=-i, \quad \gamma(2,0)=i, \\
& \gamma(2,1)=\beta, \quad \gamma(2,3)=\bar{\beta}, \quad \gamma(3,0)=-\bar{\beta}, \quad \gamma(3,1)=i, \quad \gamma(3,2)=\beta, \quad \gamma(n, n)=1, \\
& \gamma(n+4, k)=-\gamma(n, k), \quad \gamma(n, k+4)=-\gamma(n, k), \quad \gamma(8 n, k)=\gamma(0, k), \quad \gamma(n, 8 k)=\gamma(n, 0) .
\end{aligned}
$$

From (3.13), we note that

$$
\left|u_{8 n+j}\right|=\exp \left(H_{j}+\frac{1}{2} \sum_{k_{1}=0}^{8 n+j-1}\left[1+(-1)^{n+k_{1}}\right] \operatorname{Re}\left(\gamma\left(n, k_{1}\right)\right) \ln \left|V_{k_{1}}\right|\right)
$$

where

$$
H_{j}=\beta^{j} c_{6}+(-1)^{j} \bar{\beta}^{j} c_{7}+(-1)^{j} \beta^{j} c_{8}+\bar{\beta}^{j} c_{9}
$$

For $j=0$, we have

$$
\left|\mathrm{u}_{8 \mathrm{n}}\right|=\exp \left(\mathrm{H}_{0}+\ln \left|\mathrm{V}_{0}\right|-\ln \left|\mathrm{V}_{4}\right|+\ln \left|\mathrm{V}_{8}\right|-\ln \left|\mathrm{V}_{12}\right|+\cdots+\ln \left|\mathrm{V}_{8 \mathrm{n}-8}\right|-\ln \left|\mathrm{V}_{8 \mathrm{n}-4}\right|\right)=\exp \left(\mathrm{H}_{0}\right) \prod_{s=0}^{\mathrm{n}-1}\left|\frac{\mathrm{V}_{8 \mathrm{~s}}}{\mathrm{~V}_{8 \mathrm{~s}+4}}\right|
$$

One can show that there is no need for the absolute values by using the fact that

$$
v_{i}=\frac{1}{u_{i} u_{i+4}} .
$$

To find $\exp \left(\mathrm{H}_{0}\right)$, we set $\mathrm{n}=0$ in (3.14) and observe that $\left|\mathrm{u}_{0}\right|=\exp \left(\mathrm{H}_{0}\right)$. Thus

$$
u_{8 n}=u_{0} \prod_{s=0}^{n-1} \frac{v_{8 s}}{v_{8 s+4}}
$$

Clearly, $8 s=2(4 s)+0$ so that $i=0$ and $n=4 s$ in (3.6). From (3.6), we obtain

$$
\begin{aligned}
\mathrm{V}_{8 s} & =\mathrm{V}_{0}\left(\prod_{\mathrm{k}_{1}=0}^{4 s-1} \mathrm{~A}_{2 \mathrm{k}_{1}}+\frac{1}{\mathrm{~V}_{0}} \sum_{\mathrm{l}=0}^{4 s-1} \mathrm{~B}_{2 \mathrm{l}} \prod_{\mathrm{k}_{2}=\mathrm{l+1}}^{4 s-1} \mathrm{~A}_{2 \mathrm{k}_{2}}\right) \\
& =\frac{1}{\mathrm{u}_{0} \mathrm{u}_{4}}\left(\prod_{\mathrm{k}_{1}=0}^{4 s-1} \mathrm{~A}_{2 \mathrm{k}_{1}}+\mathrm{u}_{0} \mathrm{u}_{4} \sum_{\mathrm{l}=0}^{4 s-1} \mathrm{~B}_{2 l} \prod_{\mathrm{k}_{2}=\mathrm{l}+1}^{4 s-1} \mathrm{~A}_{2 \mathrm{k}_{2}}\right),
\end{aligned}
$$

using (3.15). Similarly, we have

$$
v_{8 s+4}=\frac{1}{u_{0} u_{4}}\left(\prod_{k_{1}=0}^{4 s+1} A_{2 k_{1}}+u_{0} u_{4} \sum_{l=0}^{4 s+1} B_{2 l} \prod_{k_{2}=l+1}^{4 s-1} A_{2 k_{2}}\right)
$$


so that

$$
u_{8 n}=u_{0} \prod_{s=0}^{n-1} \frac{\prod_{k_{1}=0}^{4 s-1} A_{2 k_{1}}+u_{0} u_{4} \sum_{l=0}^{4 s-1} B_{2 l} \prod_{k_{2}=l+1}^{4 s-1} A_{2 k_{2}}}{\prod_{k_{1}=0}^{4 s+1} A_{2 k_{1}}+u_{0} u_{4} \sum_{l=0}^{4 s+1} B_{2 l} \prod_{k_{2}=l+1}^{4 s+1} A_{2 k_{2}}}
$$

Hence

$$
x_{8 n-5}=x_{-5} \prod_{s=0}^{n-1} \frac{\prod_{k_{1}=0}^{4 s-1} A_{2 k_{1}}+x_{-5} x_{-1} \sum_{l=0}^{4 s-1} B_{2 l} \prod_{k_{2}=l+1}^{4 s-1} A_{2 k_{2}}}{\prod_{k_{1}=0}^{4 s+1} A_{2 k_{1}}+x_{-5} x_{-1} \sum_{l=0}^{4 s+1} B_{2 l} \prod_{k_{2}=l+1}^{4 s+1} A_{2 k_{2}}} .
$$

For $j=1$, (3.14) becomes

$$
\left|\mathrm{u}_{8 \mathrm{n}+1}\right|=\exp \left(\mathrm{H}_{1}\right) \exp \left(\ln \left|\mathrm{V}_{1}\right|-\ln \left|\mathrm{V}_{5}\right|+\ln \left|\mathrm{V}_{9}\right|-\ln \left|\mathrm{V}_{13}\right|+\cdots+\ln \left|\mathrm{V}_{8 \mathrm{n}-7}\right|-\ln \left|\mathrm{V}_{8 \mathrm{n}-3}\right|\right) .
$$

However, using (3.14) with $j=1, n=0,\left|u_{1}\right|=\exp \left(H_{1}\right)$. Thus

$$
u_{8 n+1}=u_{1} \prod_{s=0}^{n-1} \frac{v_{8 s+1}}{v_{8 s+5}}
$$

By (3.6), we have

$$
\begin{aligned}
\mathrm{V}_{8 s+1} & =\mathrm{V}_{1}\left(\prod_{\mathrm{k}_{1}=0}^{4 s-1} A_{2 \mathrm{k}_{1}+1}+\frac{1}{\mathrm{~V}_{1}} \sum_{\mathrm{l}=0}^{4 s-1} B_{2 l+1} \prod_{\mathrm{k}_{2}=\mathrm{l}+1}^{4 s-1} A_{2 \mathrm{k}_{2}}\right) \\
& =\frac{1}{\mathrm{u}_{1} \mathrm{u}_{5}}\left(\prod_{\mathrm{k}_{1}=0}^{4 s-1} A_{2 \mathrm{k}_{1}+1}+\mathrm{u}_{1} \mathrm{u}_{5} \sum_{\mathrm{l}=0}^{4 s-1} B_{2 l+1} \prod_{\mathrm{k}_{2}=\mathrm{l}+1}^{4 s-1} A_{2 \mathrm{k}_{2}+1}\right),
\end{aligned}
$$

and similarly,

$$
v_{8 s+5}=\frac{1}{u_{1} u_{5}}\left(\prod_{k_{1}=0}^{4 s+1} A_{2 k_{1}+1}+u_{1} u_{5} \sum_{l=0}^{4 s+1} B_{2 l+1} \prod_{k_{2}=l+1}^{4 s+1} A_{2 k_{2}+1}\right)
$$

Now

$$
u_{8 n+1}=u_{1} \prod_{s=0}^{n-1} \frac{\prod_{k_{1}=0}^{4 s-1} A_{2 k_{1}+1}+u_{1} u_{5} \sum_{l=0}^{4 s-1} B_{2 l+1} \prod_{k_{2}=l+1}^{4 s-1} A_{2 k_{2}+1}}{\prod_{k_{1}=0}^{4 s+1} A_{2 k_{1}+1}+u_{1} u_{5} \sum_{l=0}^{4 s+1} B_{2 l+1} \prod_{k_{2}=l+1}^{4 s+1} A_{2 k_{2}+1}},
$$

which implies that

$$
x_{8 n-4}=x_{-4} \prod_{s=0}^{n-1} \frac{\prod_{k_{1}=0}^{4 s-1} A_{2 k_{1}+1}+x_{-4} x_{0} \sum_{l=0}^{4 s-1} B_{2 l+1} \prod_{k_{2}=l+1}^{4 s-1} A_{2 k_{2}+1}}{\prod_{k_{1}=0}^{4 s+1} A_{2 k_{1}+1}+x_{-4} x_{0} \sum_{l=0}^{4 s+1} B_{2 l+1} \prod_{k_{2}=l+1}^{4 s+1} A_{2 k_{2}+1}} .
$$

For $j=2$, we find that (3.14) becomes

$$
\left|\mathrm{u}_{8 \mathrm{n}+2}\right|=\exp \left(\mathrm{H}_{2}\right) \exp \left(\ln \left|\mathrm{V}_{2}\right|-\ln \left|\mathrm{V}_{6}\right|+\ln \left|\mathrm{V}_{10}\right|-\cdots+\ln \left|\mathrm{V}_{8 \mathrm{n}-6}\right|-\ln \left|\mathrm{V}_{8 \mathrm{n}-2}\right|\right) .
$$

Similar to the earlier cases, setting $n=0$ and $j=2$ yields the equation $\left|u_{2}\right|=\exp \left(H_{2}\right)$. So we have

$$
u_{8 n+2}=u_{2} \prod_{s=0}^{n-1} \frac{v_{8 s+2}}{v_{8 s+6}}
$$

The expressions for $V_{8 s+2}$ and $V_{8 s+6}$ are obtained from (3.6), by setting $n=4 s+1, i=0$ and $n=4 s+3, i=0$, respectively. They are as follows:

$$
V_{8 s+2}=V_{0}\left(\prod_{k_{1}=0}^{4 s} A_{2 k_{1}}+\frac{1}{V_{0}} \sum_{l=0}^{4 s} B_{2 l} \prod_{k_{2}=l+1}^{4 s} A_{2 k_{2}}\right)
$$




$$
=\frac{1}{u_{0} u_{4}}\left(\prod_{k_{1}=0}^{4 s} A_{2 k_{1}}+u_{0} u_{4} \sum_{l=0}^{4 s} B_{2 l} \prod_{k_{2}=l+1}^{4 s} A_{2 k_{2}}\right)
$$

and

$$
\begin{aligned}
\mathrm{v}_{8 s+6} & =\mathrm{v}_{0}\left(\prod_{\mathrm{k}_{1}=0}^{4 s+2} A_{2 \mathrm{k}_{1}}+\frac{1}{\mathrm{~V}_{0}} \sum_{\mathrm{l}=0}^{4 s+2} \mathrm{~B}_{2 l} \prod_{\mathrm{k}_{2}=l+1}^{4 s} \mathrm{~A}_{2 \mathrm{k}_{2}}\right) \\
& =\frac{1}{\mathrm{u}_{0} \mathrm{u}_{4}}\left(\prod_{\mathrm{k}_{1}=0}^{4 s+2} \mathrm{~A}_{2 \mathrm{k}_{1}}+\mathrm{u}_{0} \mathrm{u}_{4} \sum_{\mathrm{l}=0}^{4 s+2} \mathrm{~B}_{2 l} \prod_{\mathrm{k}_{2}=\mathrm{l}+1}^{4 s+2} \mathrm{~A}_{2 \mathrm{k}_{2}}\right)
\end{aligned}
$$

Hence

$$
u_{8 n+2}=u_{2} \prod_{s=0}^{n-1} \frac{\prod_{k_{1}=0}^{4 s} A_{2 k_{1}}+u_{0} u_{4} \sum_{l=0}^{4 s} B_{2 l} \prod_{k_{2}=l+1}^{4 s} A_{2 k_{2}}}{\prod_{k_{1}=0}^{4 s+2} A_{2 k_{1}}+u_{0} u_{4} \sum_{l=0}^{4 s+2} B_{2 l} \prod_{k_{2}=l+1}^{4 s+2} A_{2 k_{2}}},
$$

which gives

$$
x_{8 n-3}=x_{-3} \prod_{s=0}^{n-1} \frac{\prod_{k_{1}=0}^{4 s} A_{2 k_{1}}+x_{-5} x_{-1} \sum_{l=0}^{4 s} B_{2 l} \prod_{k_{2}=l+1}^{4 s} A_{2 k_{2}}}{\prod_{k_{1}=0}^{4 s+2} A_{2 k_{1}}+x_{-5} x_{-1} \sum_{l=0}^{4 s+2} B_{2 l} \prod_{k_{2}=l+1}^{4 s+2} A_{2 k_{2}}} .
$$

For $j=3$, we find that (3.14) becomes

$$
\left|\mathrm{u}_{8 \mathrm{n}+3}\right|=\exp \left(\mathrm{H}_{3}\right) \exp \left(\ln \left|\mathrm{V}_{3}\right|-\ln \left|\mathrm{V}_{7}\right|+\ln \left|\mathrm{V}_{11}\right|-\cdots+\ln \left|\mathrm{V}_{8 \mathrm{n}-5}\right|-\ln \left|\mathrm{V}_{8 \mathrm{n}-1}\right|\right) .
$$

Setting $n=0$ and $j=3$, we find that $\left|u_{3}\right|=\exp \left(\mathrm{H}_{3}\right)$, hence

$$
u_{8 n+3}=u_{3} \prod_{s=1}^{n-1} \frac{v_{8 s+3}}{v_{8 s+7}}
$$

Following a similar approach as was done in the earlier cases $(j=0,1,2)$, the reader can verify that

$$
u_{8 n+3}=u_{3} \prod_{s=0}^{n-1} \frac{\prod_{k_{1}=0}^{4 s} A_{2 k_{1}+1}+u_{1} u_{5} \sum_{l=0}^{4 s} B_{2 l+1} \prod_{k_{2}=l+1}^{4 s} A_{2 k_{2}+1}}{\prod_{k_{1}=0}^{4 s+2} A_{2 k_{1}+1}+u_{1} u_{5} \sum_{l=0}^{4 s+2} B_{2 l+1} \prod_{k_{2}=l+1}^{4 s+2} A_{2 k_{2}+1}} .
$$

Thus

$$
x_{8 n-2}=x_{-2} \prod_{s=0}^{n-1} \frac{\prod_{k_{1}=0}^{4 s} A_{2 k_{1}+1}+x_{-4} x_{0} \sum_{l=0}^{4 s} B_{2 l+1} \prod_{k_{2}=l+1}^{4 s} A_{2 k_{2}+1}}{\prod_{k_{1}=0}^{4 s+2} A_{2 k_{1}+1}+x_{-4} x_{0} \sum_{l=0}^{4 s+2} B_{2 l+1} \prod_{k_{2}=l+1}^{4 s+2} A_{2 k_{2}+1}}
$$

For $j=4$, (3.14) becomes

$$
\left|\mathrm{u}_{8 \mathrm{n}+4}\right|=\exp \left(\mathrm{H}_{4}\right) \exp \left(-\ln \left|\mathrm{V}_{0}\right|+\ln \left|\mathrm{V}_{4}\right|-\ln \left|\mathrm{V}_{8}\right|+\cdots+\ln \left|\mathrm{V}_{8 \mathrm{n}-4}\right|-\ln \left|\mathrm{V}_{8 \mathrm{n}}\right|\right) .
$$

Setting $j=0$ and $n=0$ in (3.14) yields $\left|u_{4}\right|=\exp \left(H_{4}\right) \exp \left(-\ln \left|V_{0}\right|\right)$, so that $\exp \left(H_{4}\right)=\left|u_{4}\right|\left|V_{0}\right|$. Thus

$$
u_{8 n+4}=u_{4} \prod_{s=1}^{n-1} \frac{v_{8 s+4}}{v_{8 s+8}}
$$

As before, similar steps can be carried out and one obtains

$$
\mathrm{u}_{8 \mathrm{n}+4}=\mathrm{u}_{4} \prod_{s=0}^{n-1} \frac{\prod_{\mathrm{k}_{1}=0}^{4 \mathrm{~s}+1} A_{2 \mathrm{k}_{1}}+\mathrm{u}_{0} \mathrm{u}_{4} \sum_{\mathrm{l}=0}^{4 s+1} B_{2 l} \prod_{\mathrm{k}_{2}=\mathrm{l}+1}^{4 \mathrm{~s}+1} A_{2 \mathrm{k}_{2}}}{\prod_{\mathrm{k}_{1}=0}^{4 s+3} A_{2 \mathrm{k}_{1}}+\mathrm{u}_{0} \mathrm{u}_{4} \sum_{\mathrm{l}=0}^{4 s+3} \mathrm{~B}_{2 l} \prod_{\mathrm{k}_{2}=\mathrm{l}+1}^{4 s+3} A_{2 \mathrm{k}_{2}}}
$$


which yields

$$
x_{8 n-1}=x_{-1} \prod_{s=0}^{n-1} \frac{\prod_{k_{1}=0}^{4 s+1} A_{2 k_{1}}+x_{-5} x_{-1} \sum_{l=0}^{4 s+1} B_{2 l} \prod_{k_{2}=l+1}^{4 s+1} A_{2 k_{2}}}{\prod_{k_{1}=0}^{4 s+3} A_{2 k_{1}}+x_{-5} x_{-1} \sum_{l=0}^{4 s+3} B_{2 l} \prod_{k_{2}=l+1}^{4 s+3} A_{2 k_{2}}} .
$$

For $j=5$, (3.14) becomes

$$
\left|\mathrm{u}_{8 \mathrm{n}+5}\right|=\exp \left(\mathrm{H}_{5}\right) \exp \left(-\ln \left|\mathrm{V}_{1}\right|+\ln \left|\mathrm{V}_{5}\right|-\ln \left|\mathrm{V}_{9}\right|+\cdots+\ln \left|\mathrm{V}_{8 \mathrm{n}-3}\right|-\ln \left|\mathrm{V}_{8 \mathrm{n}+1}\right|\right) .
$$

But setting $n=0$ and $j=5$ in the same equation (3.14), we get $\left|u_{5}\right|=\exp \left(-\ln \left|V_{1}\right|\right) \exp \left(H_{5}\right)$ so that $\exp \left(\mathrm{H}_{5}\right)=\left|\mathrm{u}_{5}\right|\left|\mathrm{V}_{1}\right|$. Thus

$$
u_{8 n+5}=u_{5} \prod_{s=0}^{n-1} \frac{v_{8 s+5}}{v_{8 s+9}}
$$

After performing similar substitutions as before, we get

$$
u_{8 n+5}=u_{5} \prod_{s=0}^{n-1} \frac{\prod_{k_{1}=0}^{4 s+1} A_{2 k_{1}+1}+u_{1} u_{5} \sum_{l=0}^{4 s+1} B_{2 l+1} \prod_{k_{2}=l+1}^{4 s+1} A_{2 k_{2}+1}}{\prod_{k_{1}=0}^{4 s+3} A_{2 k_{1}+1}+u_{1} u_{5} \sum_{l=0}^{4 s+3} B_{2 l+1} \prod_{k_{2}=l+1}^{4 s+3} A_{2 k_{2}+1}},
$$

which leads to

$$
x_{8 n}=x_{0} \prod_{s=0}^{n-1} \frac{\prod_{k_{1}=0}^{4 s+1} A_{2 k_{1}+1}+x_{-4} x_{0} \sum_{l=0}^{4 s+1} B_{2 l+1} \prod_{k_{2}=l+1}^{4 s+1} A_{2 k_{2}+1}}{\prod_{k_{1}=0}^{4 s+3} A_{2 k_{1}+1}+x_{-4} x_{0} \sum_{l=0}^{4 s+3} B_{2 l+1} \prod_{k_{2}=l+1}^{4 s+3} A_{2 k_{2}+1}} .
$$

For $j=6$ and $n=0$, (3.14) becomes $\left|u_{6}\right|=\exp \left(H_{6}\right) \exp \left(-\ln \left|V_{2}\right|\right)$, i.e., $\exp \left(H_{6}\right)=\left|u_{6}\right|\left|V_{2}\right|$. However, setting $j=6$ in the same equation, we get

$$
\left|u_{8 n+6}\right|=\exp \left(H_{6}\right) \exp \left(-\ln \left|V_{2}\right|+\ln \left|V_{6}\right|-\ln \left|V_{10}\right|+\cdots+\ln \left|V_{8 n-2}\right|-\ln \left|V_{8 n+2}\right|\right)
$$

We have

$$
u_{8 n+6}=u_{6} \prod_{s=0}^{n-1} \frac{v_{8 s+6}}{v_{8 s+10}}
$$

The reader can verify that

$$
u_{8 n+6}=u_{6} \prod_{s=0}^{n-1} \frac{\prod_{k_{1}=0}^{4 s+2} A_{2 k_{1}}+u_{0} u_{4} \sum_{l=0}^{4 s+2} B_{2 l} \prod_{k_{2}=l+1}^{4 s+2} A_{2 k_{2}}}{\prod_{k_{1}=0}^{4 s+4} A_{2 k_{1}}+u_{0} u_{4} \sum_{l=0}^{4 s+4} B_{2 l} \prod_{k_{2}=l+1}^{4 s+4} A_{2 k_{2}}},
$$

which gives

$$
x_{8 n+1}=x_{1} \prod_{s=0}^{n-1} \frac{\prod_{k_{1}=0}^{4 s+2} A_{2 k_{1}}+x_{-5} x_{-1} \sum_{l=0}^{4 s+2} B_{2 l} \prod_{k_{2}=l+1}^{4 s+2} A_{2 k_{2}}}{\prod_{k_{1}=0}^{4 s+4} A_{2 k_{1}}+x_{-5} x_{-1} \sum_{l=0}^{4 s+4} B_{2 l} \prod_{k_{2}=l+1}^{4 s+4} A_{2 k_{2}}} .
$$

Since $x_{1}=\frac{x_{-1} x_{-5}}{x_{-3}\left(a_{0}+b_{0} x_{-1} x_{-5}\right)}$, we have

$$
x_{8 n+1}=\frac{x_{-1} x_{-5}}{x_{-3}\left(a_{0}+b_{0} x_{-1} x_{-5}\right)} \prod_{s=0}^{n-1} \frac{\prod_{k_{1}=0}^{4 s+2} A_{2 k_{1}}+x_{-5} x_{-1} \sum_{l=0}^{4 s+2} B_{2 l} \prod_{k_{2}=l+1}^{4 s+2} A_{2 k_{2}}}{\prod_{k_{1}=0}^{4 s+4} A_{2 k_{1}}+x_{-5} x_{-1} \sum_{l=0}^{4 s+4} B_{2 l} \prod_{k_{2}=l+1}^{4 s+4} A_{2 k_{2}}} .
$$

For $j=7,(3.14)$ becomes

$$
\left|\mathrm{u}_{8 \mathrm{n}+7}\right|=\exp \left(\mathrm{H}_{7}\right) \exp \left(-\ln \left|\mathrm{V}_{3}\right|+\ln \left|\mathrm{V}_{7}\right|-\ln \left|\mathrm{V}_{11}\right|+\cdots+\ln \left|\mathrm{V}_{8 \mathrm{n}-1}\right|-\ln \left|\mathrm{V}_{8 \mathrm{n}+3}\right|\right) .
$$

By setting $n=0$ and $j=7$ in (3.14), we get $\left|u_{7}\right|=\exp \left(-\ln \left|V_{3}\right|\right) \exp \left(H_{7}\right)$ so that $\exp \left(H_{7}\right)=\left|u_{7}\right|\left|V_{3}\right|$. Hence

$$
u_{8 n+7}=u_{7} \prod_{s=0}^{n-1} \frac{v_{8 s+7}}{v_{8 s+11}}
$$


After replacing $V_{i}$ 's with their appropriate expressions as done before, we get

$$
u_{8 n+7}=u_{7} \prod_{s=0}^{n-1} \frac{\prod_{k_{1}=0}^{4 s+2} A_{2 k_{1}+1}+u_{1} u_{5} \sum_{l=0}^{4 s+2} B_{2 l+1} \prod_{k_{2}=l+1}^{4 s+2} A_{2 k_{2}+1}}{\prod_{k_{1}=0}^{4 s+4} A_{2 k_{1}+1}+u_{1} u_{5} \sum_{l=0}^{4 s+4} B_{2 l+1} \prod_{k_{2}=l+1}^{4 s+4} A_{2 k_{2}+1}},
$$

which implies that

$$
x_{8 n+2}=x_{2} \prod_{s=0}^{n-1} \frac{\prod_{k_{1}=0}^{4 s+2} A_{2 k_{1}+1}+x_{-4} x_{0} \sum_{l=0}^{4 s+2} B_{2 l+1} \prod_{k_{2}=l+1}^{4 s+2} A_{2 k_{2}+1}}{\prod_{k_{1}=0}^{4 s+4} A_{2 k_{1}+1}+x_{-4} x_{0} \sum_{l=0}^{4 s+4} B_{2 l+1} \prod_{k_{2}=l+1}^{4 s+4} A_{2 k_{2}+1}} .
$$

Since $x_{2}=\frac{x_{0} x_{-4}}{x_{-2}\left(a_{1}+b_{2} x_{0} x_{-4}\right)}$, we have

$$
x_{8 n+2}=\frac{x_{0} x_{-4}}{x_{-2}\left(a_{1}+b_{1} x_{0} x_{-4}\right)} \prod_{s=0}^{n-1} \frac{\prod_{k_{1}=0}^{4 s+2} A_{2 k_{1}+1}+x_{-4} x_{0} \sum_{l=0}^{4 s+2} B_{2 l+1} \prod_{k_{2}=l+1}^{4 s+2} A_{2 k_{2}+1}}{\prod_{k_{1}=0}^{4 s+4} A_{2 k_{1}+1}+x_{-4} x_{0} \sum_{l=0}^{4 s+4} B_{2 l+1} \prod_{k_{2}=l+1}^{4 s+4} A_{2 k_{2}+1}} .
$$

Remark 3.1. It is important to make sure that the denominators in the expressions for $x_{8 n+j}$ where $j=-5,-4, \cdots, 2$ are non-zero so that the solution is well-defined.

\section{The case when $A_{j}$ and $B_{j}$ are 2-periodic sequences}

We assume that $\left\{A_{j}\right\}_{j} \geqslant 0=\left\{A_{0}, A_{1}, A_{0}, \cdots\right\}$ where $A_{0} \neq A_{1}$, and $\{B\}_{j} \geqslant 0=\left\{B_{0}, B_{1}, B_{0}, B_{1}, \cdots\right\}$ with $B_{0} \neq B_{1}$. Then after substitution, (3.16), (3.17), (3.18), (3.19), (3.20), (3.21) and (3.22) become

$$
\begin{array}{ll}
x_{8 n-5}=x_{-5} \prod_{s=0}^{n-1} \frac{A_{0}^{4 s}+x_{-5} x_{-1} B_{0} \sum_{j=0}^{4 s-1} A_{0}^{j}}{A_{0}^{4 s+2}+x_{-5} x_{-1} B_{0} \sum_{j=0}^{4 s+1} A_{0}^{j},} & x_{8 n-4}=x_{-4} \prod_{s=0}^{n-1} \frac{A_{1}^{4 s}+x_{-4} x_{0} B_{1} \sum_{j=0}^{4 s-1} A_{1}^{j}}{A_{1}^{4 s+2}+x_{-4} x_{0} B_{1} \sum_{j=0}^{4 s+1} A_{1}^{j},} \\
x_{8 n-3}=x_{-3} \prod_{s=0}^{n-1} \frac{A_{0}^{4 s+1}+x_{-5} x_{-1} B_{0} \sum_{j=0}^{4 s} A_{0}^{j}}{A_{0}^{4 s+3}+x_{-5} x_{-1} B_{0} \sum_{j=0}^{4 s+2} A_{0}^{j}}, & x_{8 n-2}=x_{-2} \prod_{s=0}^{n-1} \frac{A_{1}^{4 s+1}+x_{-4} x_{0} B_{1} \sum_{j=0}^{4 s} A_{1}^{j}}{A_{1}^{4 s+3}+x_{-4} x_{0} B_{1} \sum_{j=0}^{4 s+2} A_{1}^{j},} \\
x_{8 n-1}=x_{-1} \prod_{s=0}^{n-1} \frac{A_{0}^{4 s+2}+x_{-5} x_{-1} B_{0} \sum_{j=0}^{4 s+1} A_{0}^{j}}{A_{0}^{4 s+4}+x_{-5} x_{-1} B_{0} \sum_{j=0}^{4 s+3} A_{0}^{j}}, & x_{8 n}=x_{0} \prod_{s=0}^{n-1} \frac{A_{1}^{4 s+2}+x_{-4} x_{0} B_{1} \sum_{j=0}^{4 s+1} A_{1}^{j}}{A_{1}^{4 s+4}+x_{-4} x_{0} B_{1} \sum_{j=0}^{4 s+3} A_{1}^{j},} \\
x_{8 n+1}=x_{1} \prod_{s=0}^{n-1} \frac{A_{0}^{4 s+3}+x_{-5} x_{-1} B_{0} \sum_{j=0}^{4 s+2} A_{0}^{j}}{A_{0}^{4 s+5}+x_{-5} x_{-1} B_{0} \sum_{j=0}^{4 s+4} A_{0}^{j},} & x_{8 n+2}=x_{2} \prod_{s=0}^{n-1} \frac{A_{1}^{4 s+3}+x_{-4} x_{0} B_{1} \sum_{j=0}^{4 s+2} A_{1}^{j}}{A_{1}^{4 s+5}+x_{-4} x_{0} B_{1} \sum_{j=0}^{4 s+4} A_{1}^{j},}
\end{array}
$$

where $x_{1}=\frac{x_{1} x_{-5}}{x_{-3}\left(A_{0}+B_{0} x_{-1} x_{-5}\right)}$ and $x_{2}=\frac{x_{0} x_{-4}}{x_{-2}\left(A_{1}+B_{1} x_{0} x_{-4}\right)}$.

The solution is only valid when the denominator in each of the solution equations above is non-zero. To state this precisely, define $h(s, j, i)$, for $i=0,1$, as follows:

$$
h(s, j, i)= \begin{cases}\frac{A_{i}^{4 s+j-1}\left(1-A_{i}\right)}{B_{i}\left(1-A_{i}^{4 s+j+1}\right)}, & A_{i} \neq 1 \\ \frac{1}{B_{i}(4 s+j+1)}, & A_{i}=1 .\end{cases}
$$

Thus the solution is well-defined if

$$
x_{-2} x_{-3}\left(x_{-4} x_{0}+\frac{A_{1}}{B_{1}}\right)\left(x_{-1} x_{-5}+\frac{A_{0}}{B_{0}}\right) \prod_{j=1}^{4}\left(x_{-4} x_{0}+h(s, j, 1)\right)\left(x_{-1} x_{-5}+h(s, j, 0)\right) \neq 0,
$$

for all $s=1,2, \cdots, n-1$. 


\section{The case where $A_{j}$ and $B_{j}$ are 1-periodic sequences}

In this setting we assume that $A_{j}=A_{0}$, and $B_{j}=B_{0}$ for all $j$. The solution is found by replacing $A_{1}$ and $B_{1}$ with $A_{0}$ and $B_{0}$, respectively in the solution equations for Section 4 . We find the following solution equations:

$$
\begin{aligned}
& x_{8 n-5}=x_{-5} \prod_{s=0}^{n-1} \frac{A_{0}^{4 s}+x_{-5} x_{-1} B_{0} \sum_{j=0}^{4 s-1} A_{0}^{j}}{A_{0}^{4 s+2}+x_{-5} x_{-1} B_{0} \sum_{j=0}^{4 s+1} A_{0}^{j}}, \quad x_{8 n-4}=x_{-4} \prod_{s=0}^{n-1} \frac{A_{0}^{4 s}+x_{-4} x_{0} B_{0} \sum_{j=0}^{4 s-1} A_{0}^{j}}{A_{0}^{4 s+2}+x_{-4} x_{0} B_{0} \sum_{j=0}^{4 s+1} A_{0}^{j}}, \\
& x_{8 n-3}=x_{-3} \prod_{s=0}^{n-1} \frac{A_{0}^{4 s+1}+x_{-5} x_{-1} B_{0} \sum_{j=0}^{4 s} A_{0}^{j}}{A_{0}^{4 s+3}+x_{-5} x_{-1} B_{0} \sum_{j=0}^{4 s+2} A_{0}^{j}}, \quad x_{8 n-2}=x_{-2} \prod_{s=0}^{n-1} \frac{A_{0}^{4 s+1}+x_{-4} x_{0} B_{0} \sum_{j=0}^{4 s} A_{0}^{j}}{A_{0}^{4 s+3}+x_{-4} x_{0} B_{0} \sum_{j=0}^{4 s+2} A_{0}^{j}}, \\
& x_{8 n-1}=x_{-1} \prod_{s=0}^{n-1} \frac{A_{0}^{4 s+2}+x_{-5} x_{-1} B_{0} \sum_{j=0}^{4 s+1} A_{0}^{j}}{A_{0}^{4 s+4}+x_{-5} x_{-1} B_{0} \sum_{j=0}^{4 s+3} A_{0}^{j}}, \quad x_{8 n}=x_{0} \prod_{s=0}^{n-1} \frac{A_{0}^{4 s+2}+x_{-4} x_{0} B_{0} \sum_{j=0}^{4 s+1} A_{0}^{j}}{A_{0}^{4 s+4}+x_{-4} x_{0} B_{0} \sum_{j=0}^{4 s+3} A_{0}^{j}} \\
& x_{8 n+1}=x_{1} \prod_{s=0}^{n-1} \frac{A_{0}^{4 s+3}+x_{-5} x_{-1} B_{0} \sum_{j=0}^{4 s+2} A_{0}^{j}}{A_{0}^{4 s+5}+x_{-5} x_{-1} B_{0} \sum_{j=0}^{4 s+4} A_{0}^{j}}, \quad x_{8 n+2}=x_{2} \prod_{s=0}^{n-1} \frac{A_{0}^{4 s+3}+x_{-4} x_{0} B_{0} \sum_{j=0}^{4 s+2} A_{0}^{j}}{A_{0}^{4 s+5}+x_{-4} x_{0} B_{0} \sum_{j=0}^{4 s+4} A_{0}^{j}},
\end{aligned}
$$

where $x_{1}=\frac{x_{1} x_{-5}}{x_{-3}\left(A_{0}+B_{0} x_{-1} x_{-5}\right)}$ and $x_{2}=\frac{x_{0} x_{-4}}{x_{-2}\left(A_{0}+B_{0} x_{0} x_{-4}\right)}$.

We also notice that the solution is well-defined if

$$
x_{-2} x_{-3}\left(x_{-4} x_{0}+\frac{A_{0}}{B_{0}}\right)\left(x_{-1} x_{-5}+\frac{A_{0}}{B_{0}}\right) \prod_{j=1}^{4}\left(x_{-4} x_{0}+h(s, j, 0)\right)\left(x_{-1} x_{-5}+h(s, j, 0)\right) \neq 0
$$

for all $s=1,2, \cdots, n-1$, where the definition of $h(s, j, i)$ is given in the previous section.

5.1. The case of $A_{j}=B_{j}=-1$

In this case, set $A_{0}=B_{0}=-1$ in the above equations. This yields the following solution equations, which appear in [9, Theorem 5.1].

$$
\begin{array}{llrl}
x_{8 n-5}=x_{-5}, & x_{8 n-4}=x_{-4} & x_{8 n-3}=x_{-3}, \\
x_{8 n-2}=x_{-2}, & x_{8 n-1}=x_{-1}, & x_{8 n}=x_{0}, \\
x_{8 n+1}=\frac{x_{1} x_{-5}}{x_{-3}\left(-1-x_{-1} x_{-5}\right)}, & x_{8 n+2}=\frac{x_{0} x_{-4}}{x_{-2}\left(-1-x_{0} x_{-4}\right)} . &
\end{array}
$$

5.2. The case of $A_{j}=-1$ and $B_{j}=1$

This case yields the following solution equations which appear in [9, Theorem 3.1].

$$
\begin{array}{llrl}
x_{8 n-5}=x_{-5}, & x_{8 n-4}=x_{-4} & x_{8 n-3}=x_{-3}, \\
x_{8 n-2}=x_{-2}, & x_{8 n-1}=x_{-1}, & x_{8 n}=x_{0}, \\
x_{8 n+1}=\frac{x_{1} x_{-5}}{x_{-3}\left(-1+x_{-1} x_{-5}\right)}, & x_{8 n+2}=\frac{x_{0} x_{-4}}{x_{-2}\left(-1+x_{0} x_{-4}\right)} .
\end{array}
$$

5.3. The case of $A_{j}=1$ and $B_{j}=-1$

Our general solution yields the following solution equations which appear in [9, Theorem 4.1].

$$
\begin{array}{ll}
x_{8 n-5}=x_{-5} \prod_{s=0}^{n-1} \frac{1-x_{-5} x_{-1} 4 s}{1-x_{-5} x_{-1}(4 s+2)}, & x_{8 n-4}=x_{-4} \prod_{s=0}^{n-1} \frac{1-x_{-4} x_{0} 4 s}{1-x_{-4} x_{0}(4 s+2)}, \\
x_{8 n-3}=x_{-3} \prod_{s=0}^{n-1} \frac{1-x_{-5} x_{-1}(4 s+1)}{1-x_{-5} x_{-1}(4 s+3)}, & x_{8 n-2}=x_{-2} \prod_{s=0}^{n-1} \frac{1-x_{-4} x_{0}(4 s+1)}{1-x_{-4} x_{0}(4 s+3)},
\end{array}
$$




$$
\begin{array}{ll}
x_{8 n-1}=x_{-1} \prod_{s=0}^{n-1} \frac{1-x_{-5} x_{-1}(4 s+2)}{1-x_{-5} x_{-1}(4 s+4)}, & x_{8 n}=x_{0} \prod_{s=0}^{n-1} \frac{1-x_{-4} x_{0}(4 s+2)}{1-x_{-4} x_{0}(4 s+4)} \\
x_{8 n+1}=x_{1} \prod_{s=0}^{n-1} \frac{1-x_{-5} x_{-1}(4 s+3)}{1-x_{-5} x_{-1}(4 s+5)}, & x_{8 n+2}=x_{2} \prod_{s=0}^{n-1} \frac{1-x_{-4} x_{0}(4 s+3)}{1-x_{-4} x_{0}(4 s+5)}
\end{array}
$$

where $x_{1}=\frac{x_{1} x_{-5}}{x_{-3}\left(1-x_{-1} x_{-5}\right)}$ and $x_{2}=\frac{x_{0} x_{-4}}{x_{-2}\left(1-x_{0} x_{-4}\right)}$.

5.4. The case of $A_{j}=B_{j}=1$

This case yields the following solution equations which appear in [9, Theorem 2.1].

$$
\begin{array}{rlrl}
x_{8 n-5} & =x_{-5} \prod_{s=0}^{n-1} \frac{1+x_{-5} x_{-1} 4 s}{1+x_{-4} x_{0}(4 s+2)}, & x_{8 n-4}=x_{-4} \prod_{s=0}^{n-1} \frac{1+x_{-4} x_{0} 4 s}{1+x_{-4} x_{0}(4 s+2)}, \\
x_{8 n-3}=x_{-3} \prod_{s=0}^{n-1} \frac{1+x_{-5} x_{-1}(4 s+1)}{1+x_{-5} x_{-1}(4 s+3)}, & x_{8 n-2}=x_{-2} \prod_{s=0}^{n-1} \frac{1+x_{-4} x_{0}(4 s+1)}{1+x_{-4} x_{0}(4 s+3)} \\
x_{8 n-1}=x_{-1} \prod_{s=0}^{n-1} \frac{1+x_{-5} x_{-1}(4 s+2)}{1+x_{-5} x_{-1}(4 s+4)}, & x_{8 n}=x_{0} \prod_{s=0}^{n-1} \frac{1+x_{-4} x_{0}(4 s+2)}{1+x_{-4} x_{0}(4 s+4)}, \\
x_{8 n+1}=x_{1} \prod_{s=0}^{n-1} \frac{1+x_{-5} x_{-1}(4 s+3)}{1+x_{-5} x_{-1}(4 s+5)}, & x_{8 n+2}=x_{2} \prod_{s=0}^{n-1} \frac{1+x_{-4} x_{0}(4 s+3)}{1+x_{-4} x_{0}(4 s+5)},
\end{array}
$$

where $x_{1}=\frac{x_{1} x_{-5}}{x_{-3}\left(1+x_{-1} x_{-5}\right)}$ and $x_{2}=\frac{x_{0} x_{-4}}{x_{-2}\left(1+x_{0} x_{-4}\right)}$.

\section{Conclusion}

In this paper, by using a symmetry based method, we have obtained a solution of a more general form of the difference equations considered in [9]. Thus we studied difference equations of the form

$$
x_{n+1}=\frac{x_{-1} x_{n-5}}{x_{n-3}\left(A_{n}+B_{n} x_{n-1} x_{n-5}\right)}
$$

and obtained their solutions. It should be noted that Khaliq and Elsayed [9] considered the cases with all possible combinations of $A_{n} \in\{-1,1\}$ and $B_{n} \in\{-1,1\}$, and our case goes beyond the \pm 1 setting by generalizing the situation to $\left(B_{n}\right)$ and $\left(A_{n}\right)$ being non-zero sequences of real numbers.

\section{Acknowledgment}

We would like to thank the reviewers for their fruitful suggestions that helped improve the paper.

\section{References}

[1] E. M. Elsayed, T. F. Ibrahim, Periodicity and solutions for some systems of nonlinear rational difference equations, Hacet. J. Math. Stat., 44 (2015), 1361-1390. 1

[2] G. Feng, X.-J. Yang, H. M. Srivasta, Exact traveling-wave solutions for linear and nonlinear heat-transfer equations, Therm. Sci., 00 (2016), 6 pages. 1

[3] M. Folly-Gbetoula, Symmetry, reductions and exact solutions of the difference equation $u_{n+2}=a u_{n} /\left(1+b u_{n} u_{n+1}\right)$, J. Difference Equ. Appl., 23 (2017), 1017-1024. 1

[4] M. Folly-Gbetoula, A. H. Kara, Symmetries, conservation laws, and 'integrability' of difference equations, Adv. Difference Equ., 2014 (2014), 14 pages.

[5] P. E. Hydon, Symmetries and first integrals of ordinary difference equations, R. Soc. Lond. Proc. Ser. A Math. Phys. Eng. Sci., 456 (2000), 2835-2855. 
[6] P. E. Hydon, Difference equations by differential equation methods, Cambridge Monographs on Applied and Computational Mathematics, Cambridge University Press, Cambridge, (2014). 1, 2

[7] T. F. Ibrahim, Solving a class of three-order max-type difference equations, Dyn. Contin. Discrete Impuls. Syst. Ser. A Math. Anal., 21 (2014), 333-342. 1

[8] T. F. Ibrahim, M. A. El-Moneam, Global stability of a higher-order difference equation, Iran. J. Sci. Technol. Trans. A Sci., 41 (2017), 51-58. 1

[9] A. Khaliq, E. M. Elsayed, The dynamics and solution of some difference equations, J. Nonlinear Sci. Appl., 9 (2016), 1052-1063. 1, 5.1, 5.2, 5.3, 5.4, 6

[10] A. Q. Khan, Q. Din, M. N. Qureshi, T. F. Ibrahim, Global behavior of an anti-competitive system of fourth-order rational difference equations, Comput. Ecol. Softw., 4 (2014), 35-46. 1

[11] D. Levi, L. Vinet, P. Winternitz, Lie group formalism for difference equations, J. Phys. A, 30 (1997), 633-649. 1

[12] S. Lie, Classification und Integration von gewhnlichen Differentialgleichungen zwischen $x y$, die eine Gruppe von Transformationen gestatten, (German) Math. Ann., 22 (1888), 213-281. 1

[13] R. Morris, A. H. Kara, A. Chowdhury, A. Biswas, Soliton solutions, conservation laws, and reductions of certain classes of nonlinearwave equations, Z. Naturforsch. A, 67 (2012), 613-620. 1

[14] E. Noether, Invariante variationsprobleme, (German) Nachr. D. König. Gesellsch. D. Wiss. Zu Göttingen, Math-phys. Klasse, 1918 (1918), 235-257. 1

[15] G. R. W. Quispel, R. Sahadevan, Lie symmetries and the integration of difference equations, Phys. Lett. A, 184 (1993), 64-70. 1

[16] W. Sarlet, F. Cantrijn, Generalizations of Noether's theorem in classical mechanics, SIAM Rev., 23 (1981), 467-494. 1

[17] X.-J. Yang, F. Gao, H. M. Srivastava, Exact traveling wave solutions for the local fractional two-dimensional Burgers-type equations, Comput. Math. Appl., 73 (2017), 203-210. 1

[18] X.-J. Yang, J. A. Tenreiro Machado, D. Baleanu, Exact traveling-wave solution for local fractional Boussinesq equation in fractal domain, Fractals, 25 (2017), 7 pages. 1

[19] X.-J. Yang, J. A. Tenreiro Machado, D. Baleanu, C. Cattani, On exact traveling-wave solutions for local fractional Korteweg-de Vries equation, Chaos, 26 (2016), 5 pages. 1 